This is the final peer-reviewed accepted manuscript of:

Comparison of different filtering strategies to reduce noise in strain measurement with Digital Image Correlation

Jacopo Baldoni, Giacomo Lionello, Fabiana Zama, Luca Cristofolini

J Strain Analysis for Engineering Design 2016: 51(6): 416-430

The final published version is available online at:

https://doi.org/10.1177/0309324716646690

Rights / License:

The terms and conditions for the reuse of this version of the manuscript are specified in the publishing policy. For all terms of use and more information see the publisher's website.

This item was downloaded from IRIS Università di Bologna (https://cris.unibo.it))

When citing, please refer to the published version. 


\title{
Comparison of different filtering strategies to reduce noise in strain measurement with Digital Image Correlation
}

\author{
Jacopo Baldoni, MEng ${ }^{1}$, Giacomo Lionello, MEng ${ }^{2}$, Fabiana Zama, $\mathrm{PhD}^{3}$, \\ Luca Cristofolini, $\mathrm{PhD}^{1}$ \\ ${ }^{1}$ Department of Industrial Engineering, Alma Mater Studiorum - Università di \\ Bologna, Italy \\ ${ }^{2}$ Laboratory for Medical Technology, Rizzoli Orthopaedic Institute, Bologna, Italy \\ ${ }^{3}$ Department of Mathematics, Alma Mater Studiorum - Università di Bologna, Italy
}

Running head: Filtering strategies to reduce noise in DIC-measured strain

Submitted to: The Journal of Strain Analysis for Engineering Design

Version. 0: $\quad 7^{\text {th }}$ October 2015

Version.1: $\quad 10^{\text {th }}$ February 2016

Statistics:

Word count (manuscript): 6049 (Introduction through acknowledgements, excluding references, captions and tables)

Word count (abstract): $\quad 289$ words

Figures: 11

Tables: 4

References: 33

\section{Corresponding author:}

Luca Cristofolini

Department of Industrial Engineering, University of Bologna

Viale Risorgimento, 2 - 40136 Bologna, Italy; e-mail: luca.cristofolini@unibo.it 


\section{ABSTRACT}

The main limitation of Digital Image Correlation (DIC) is the remarkable noise affecting the DIC-computed strain distributions. Neither manufacturers of DIC systems, nor the literature provide guidelines for optimal filtering of DIC strain distributions. However, filtering is also associated with loss of information (smoothing of the strain gradients). We systematically explored different filtering strategies to reduce noise while minimizing the loss of information in the DIC-computed strain distributions. The first filtering strategy was directly applied to the acquired images that were then fed to the DIC software. Median adaptive low-pass filters, and notch filters were used to eliminate noise: both strategies increased (rather than reducing) the noise in the DIC-computed strain distributions. The second strategy explored was a Gaussian low-pass filtering of the strain distributions. When the optimal cutoff frequency was selected, the noise was remarkably reduced (by 70\%) without excessive loss of information. At the same time, when non-optimal cutoff frequencies were used, the residual noise and/or loss of information seriously compromised the results. Finally, image combination techniques were applied both to the input images, and to the strain distributions. This strategy was extremely time-consuming but not very effective (noise reduction $<10 \%$ ). In conclusion, the only truly effective noise reduction strategy, if measurements are carried out using a commercial closed software, consists in filtering the strain distribution.

Keywords: digital image correlation (DIC), strain measurement, noise reduction, loss of information, filtering strategy, optimal cutoff frequency, spatial frequency domain, adaptive filtering 


\section{INTRODUCTION}

Digital Image Correlation (DIC) has become one of the most promising non-contact strain measurement techniques. In the 3D implementation, the DIC algorithm is based on the comparison of different pairs of stereoscopic images taken at different stages of time (corresponding to different load and strain conditions), with a pair of images used as a reference (typically corresponding to the unloaded condition).

The DIC process can be divided into four main operations [1]:

1. Image acquisition;

2. Triangulation and $3 \mathrm{D}$ reconstruction;

3. Correlation and computation of displacements and strains: strain measurements carried out via DIC are the result of the minimization of a correlation function that connects the brightness distribution functions of a subset of pixels in the images of the deformed and non-deformed specimens [2];

4. Post-processing and presentation of the $3 \mathrm{D}$ displacement and strain results.

The DIC-computed displacements (to a limited extent) and strains (to a larger extent) are affected by noise [3]. The displacement field in most cases can be measured with sub-pixel accuracy and negligible noise using a commercially available DIC system $[4,5]$. The same does not apply to strain measurements, due to the derivation process that tends to emphasize noise [6]. 
Even when strain measurements are carried out on an unstrained specimen a noise pattern, sometimes exceeding $150 \times 10^{-6}$ affects the DIC-computed strains, and is superimposed to the actual strain distribution $[2,7,8]$ (Figure 1). Most of the times noise appears as a non-periodic strain pattern hiding the real gradients, some others as a periodic pattern [3]. The causes of such noise are connected to different phenomena: noise deriving from the quality of the input images (associated with irregular illumination of the specimen, camera-induced noise in the acquired images, air turbulence, unsuitable speckle size), and noise related to the correlation process (inaccurate system calibration, numerical inaccuracies in the calculation software) $[1,3]$. The random component of the error connected to the image acquisition with charge coupled device (CCD) cameras, is caused by the concurrence of a number of factors including:

- Dark noise: it arises from the statistical variation of the number of electrons thermally generated within the silicon structure of the CCD of digital cameras. This number is highly dependent on the device temperature. Dark current refers to the generation rate of thermal electrons at a given temperature [9], [10].

- Photon noise: it derives from the temporal fluctuations of the photons reaching each pixel of the sensor at each instant in time [10].

- Read noise: this is caused by the electronics connected, when reading the electrical charge collected by the sensor [10]. 
On the other hand, non-random noise creates periodic patterns in the strain distributions. Usually it is referred to as aliasing, and it is related to the impossibility to represent excessively fine speckle patterns in the images acquired [11].

In this paper we focus on noise reduction and more generally on the management of measurement error in applications where the strain to be measured ranges from 0 to a maximum of $5000 \times 10^{-6}$. This is typical of stiff polymers and many biomechanical applications (e.g. bone tissue fails when strain exceeds $7000 \times 10^{-6}$; physiological strain in bone is about $\left.1000 \times 10^{-6}[12]\right)$. It is clear that in such cases, a noise of about $150 \times 10^{-6}$ compromises the applicability of DIC as it prevents accurately detecting the real strain distribution [3].

Although an extensive literature on noise filtering exists in the field of image processing, there are few studies addressing the methods to reduce noise either filtering the input images $[13,14]$ or the DIC-computed strain distributions [15]. User manuals of commercial DIC systems suggest the iterative use of average and median smoothing filters in order to reach the expected strain distributions [16,17,18,11]. Most of the existing studies about noise reduction and accuracy enhancement focus on shape functions and sub-pixel image correlation methods used to achieve sub-pixel accuracy in strain and displacement measurements [19,20,21,22,23] or explore the chances of filtering displacement fields and then reintroduce the filtered displacements in the calculation routine of the software [24,25,26,27]. These approaches are based on customized routines and therefore cannot be implemented when using a closed 
commercial software package. Other studies emphasize the relationship between the optimization of speckle patterns and measurement noise [28,29,30]

An alternative option is filtering the input images to remove the noise due to the image acquisition system, in order to reduce the propagation of noise to the computed strain distributions. In addition, the use of different filters in the spatial frequency domain, and image combining techniques has not been thoroughly explored for DIC applications.

The aim of the current study was to systematically explore a wide range of filtering strategies to reduce noise in strain measurements carried out using a commercially available software, quantify the noise reduction achieved and the associated loss of information.

\section{COMMON MATERIALS AND METHODS}

\subsection{EXPERIMENTAL SETUP AND SPECIMENS}

The DIC measurements were carried out with a DIC 3D system (Mod. ARAMIS, GOM $\mathrm{mbH}$, Braunschweig, Germany) consisting of:

- Two 5 megapixel cameras (TXG50i, Baumer Optronic GmbH, Radeberg, Germany, Table 1) with a CCD sensor (ICX625ALA, Sony, Japan, 2448×2050 pixel resolution, Table 2) 
- Two lenses with a fixed focal length of 50mm (Mod. 2.8/50, TitanAR, Maumelle, Arkansas, USA) with circular polarizers (Jos-Schneider OptischeWerke GmbH, Bad-Kreuznach, Germany);

- Two LED lights (650lumen each);

- A $250 \mathrm{~mm} \times 200 \mathrm{~mm}$ calibration object (Mod. CP/20, GOM mbH, Braunschweig, Germany);

- A data logger (ARAMIS Sensor Controller, GOM) for power supply of the cameras, image recording, and synchronous acquisition of the analog signals;

- A high-performance PC running the DIC software (ARAMIS v6.0, GOM)

Part of the present methods and results are specific to the Aramis system we used. However, as (i) this is one of the most common commercial systems, and (ii) most commercial systems deploy similar algorithms, our observations can be extended to the majority of DIC software.

Aramis software calculates strain by tracking the variation of the $3 \mathrm{D}$ coordinates of points identified thanks to the speckle pattern. Variations of $3 \mathrm{D}$ points coordinates (displacements) from the original configuration are calculated for the bi-dimensional plane tangential to the surface of interest. It is the possible to the derive normal and shear strains (Eq. 1): 


$$
\begin{gathered}
\varepsilon_{x x}=\frac{\partial u}{\partial x}+\frac{1}{2}\left[\left(\frac{\partial u}{\partial x}\right)^{2}+\left(\frac{\partial v}{\partial x}\right)^{2}+\left(\frac{\partial w}{\partial x}\right)^{2}\right] \\
\varepsilon_{y y}=\frac{\partial v}{\partial y}+\frac{1}{2}\left[\left(\frac{\partial u}{\partial y}\right)^{2}+\left(\frac{\partial v}{\partial y}\right)^{2}+\left(\frac{\partial w}{\partial y}\right)^{2}\right] \\
\varepsilon_{x y}=\frac{1}{2}\left[\frac{\partial u}{\partial y}+\frac{\partial v}{\partial x}+\left(\frac{\partial u}{\partial x} \frac{\partial u}{\partial y}\right)+\left(\frac{\partial v}{\partial x} \frac{\partial v}{\partial y}\right)+\left(\frac{\partial w}{\partial x} \frac{\partial w}{\partial y}\right)\right]
\end{gathered}
$$

The 3D points creation process starts from the definition of appropriate correlation parameters that divide the speckle pattern in multiple subsets of a certain dimension of pixels ("facet size" parameter) partially overlapping ("facet step" parameter). The matrix of surrounding points monitored through the strain calculation process of every 3D point influences noise levels and sensitivity (computation size parameter).

Correlation parameters of the displacement and strain calculation were chosen during a preliminary evaluation of every test and followed the guidelines of specific literature [1] In order to explore the time-dependent causes of noise, the images composing every experiment were recorded with a fixed frame rate of $1 \mathrm{~Hz}$, if not differently specified. Strain measurements were made on different specimens in order to investigate different load and illumination conditions.

1. To investigate the behaviour of the DIC system with a uniform strain distribution up to relatively high strain values, a plain plate made of Polytetrafluoroethylene (PTFE measuring $270 \mathrm{~mm} \times 80 \mathrm{~mm} \times 7 \mathrm{~mm}$, with a free gage length of $170 \mathrm{~mm}$ ) was subjected to a tensile test (Figure 2a). This material was chosen because of its low Young's modulus (0.4-0.6GPa [31]) and high 
tensile elongation. These properties made it suitable for tests on a wide range of strain values. The test was performed on a servo-hydraulic testing machine (Mod.8502, Instron, Canton, MA, USA), which was fine-tuned so that actuator drift and noise did not exceed 0.1 micrometer.

2. A $20 \mathrm{~mm}$ diameter cylindrical aluminum bar (Figure $2 \mathrm{~b}$ ) was used to determine the performances of the DIC system in the non-strained condition.

3. A $360 \mathrm{~mm} \times 100 \mathrm{~mm} \times 5 \mathrm{~mm}$ plain aluminum plate was used in a cantilever arrangement to generate a known strain gradient, in order to measure the corresponding strain distributions at different deflections at the free end (Figure 2c). This was possible using a $0.75 \mathrm{~mm}$ diameter nylon wire fixed to the symmetry plane of the plate at $350 \mathrm{~mm}$ from the fixed end. The controlled traction of the wire created the desired deflection of the plate. The fixed end of the plate was connected to a rigid aluminum frame, which integrated a sliding system of Plexiglas supports to place a maximum of seven LVDTs (D5/40-AWL10, RDP, Wolverhampton, UK; and GHSA-750-500, Macro-Sensors, Pennsauken, NJ, USA) at different distances from the fixed end of the plate (Figure 2d). The class of accuracy of the LVDT sensors was 0.1 ; their signal was acquired by the ARAMIS Sensor Controller, synchronously with the images. In this way, the displacements measured by the LVDTs could be used as a reference to test the accuracy of the DIC measurements. 
A high-contrast, random and homogeneous speckle pattern was necessary for a complete description of the strain field on the surface of all the specimens. The technique adopted for the pattern creation met both the ARAMIS practical specifications and theoretical considerations [16,32]. A white water-based paint (Aquamax Extra Basecoat, MaxMeyer, PPG Italy Sales \& Services, Milano, Italy) was used for the background. Black acrylic paint was used for the speckles (Base-DS, RAL 9011, River Srl, Italy). Both paints were sprayed using an air gun brush, which was adjusted to produce speckles of the recommended size (figure 2e). All the filtering and analyses were implemented using MATLABC (2009 Edition, MathWorks, Natick, MA, USA).

\subsection{FILTERING ROUTINES IN COMMERCIAL DIC SOFTWARE}

Filtering routines based on linear filters are usually available in commercial DIC software. These filters are obtained by convolution of a filter mask $w(s, t)$ of dimension $m \times n$ (where $\mathrm{m}=2 \mathrm{a}+1, \mathrm{n}=2 \mathrm{~b}+1$ and $\mathrm{a}, \mathrm{b}>0$ ) over a data matrix $\mathrm{f}$ with dimensions $\mathrm{M} \times \mathrm{N}$, following the expression:

$$
g(x, y)=\sum_{s=-a}^{a} \sum_{t=-b}^{b} w(s, t) f(x+s, y+t), \quad x=0, \ldots M-1, \quad y=0, \ldots N-1
$$

Different filters are obtained by changing the coefficients and size of the mask [18]. Averaging masks, or weighted averaging masks, replace every value of the data matrix with the average (or weighted average) of the values in the neighborhood defined by the 
filter mask. The only options available for the user typically are: increasing the size of the mask, or applying the same filtering mask several times. This causes also loss of information at the edges and borders of the filtered data. These typologies are not suitable for impulsive noise and can lead to uncontrolled loss of information even with random noise. Moreover the user does not have any optimization criteria available.

\subsection{ERROR MEASUREMENT}

The distance between the strain values computed by the DIC software and the theoretical strain value was measured by the root mean square error (RMSE):

$$
R M S E=\sqrt{\frac{1}{n} \frac{1}{m} \sum_{i=1}^{n} \sum_{j=1}^{m}\left(\varepsilon_{D I C_{i, j}}-\hat{\varepsilon}_{i, j}\right)^{2}} \text { (Eq. 3) }
$$

where:

- $n \times m$ are the number of strain points computed by DIC;

- $\quad$ is the local strain along one dimension determined by the DIC software;

- is the corresponding theoretical strain value. The theoretical strain distribution is either null in case of non-strained specimens or has to be determined using a different measuring technique more reliable then DIC (e.g. based on the LVDT measurements). 


\subsection{APPLICATION OF THE DISCRETE FOURIER TRANSFORM}

A spectral approach was requested for the majority of the filtering methods proposed in this paper. The discrete Fourier transform (DFT) is the foundation for this approach. Details about the properties of DFT and their usage in image processing can be found in [33]. In order to filter either the specimen images, or the computed strain distributions, the bi-dimensional extension of the DFT is needed. The DFT of a function (an image, or a punctual strain distribution of $\mathrm{Mx} \mathrm{N}$ size) is given by:

(Eq. 4)

The Fourier amplitude spectrum is defined as:

$$
(F(u, v))=\left[R^{2}(u, v)+I^{2}(u, v)\right]^{1 / 2}(\text { Eq.5) }
$$

where:

- $\quad R$ and $I$ are respectively the real and imaginary parts of $F(u, v)$.

All the representations of Fourier spectra are obtained multiplying the input function by $(-1)^{x+y}$ prior to computing the Fourier transform, in order to locate the origin of the Fourier transform $F(0,0)$ in the centre of the $\mathrm{M} \times \mathrm{N}$ area covered by the 2-dimensional DFT. Practical details about the implementation of such filters can be found in [18]. 


\subsection{DIGITAL IMAGE FILTERS}

Both spatial and frequency domain filters were tested in the present work.

In the spatial domain, median adaptive filters (edge preserving smoothing nonlinear filters) were implemented. They represent an improvement of median filters since they can better distinguish fine detail from noise. Median adaptive filters are used in image processing in order to remove noise, while preserving details from blurring. The median adaptive filter identifies pixels as noisy by comparing each pixel in the image to its surrounding neighbor pixels. The size of the neighborhood (filter mask) is adjustable, as well as the threshold for the comparison. The noise pixels are replaced by the median value of non-noise pixels in the neighborhood.

In the spatial frequency domain, Gaussian low-pass filters as well as Notch filters were implemented. Gaussian low-pass filters were used to remove high-frequency components from Fourier spectra, and to control the ringing artifacts produced by ideal filters. The transfer function of the Gaussian low-pass filter implemented $\mathrm{H}$ was:

$$
H(u, v)=e^{-D^{2}(u, v) / 2 D_{0}^{2}}(\text { Eq. 6) }
$$

where:

- $\quad$ is the cutoff frequency of the filter that controls the shape of the Gaussian;

- $D(u, v)$ is the distance of point $(u, v)$ from the centre of the spectrum. 
When $D(u, v)=D_{0}$ the filter decreases by 0.607 the maximum value of the frequency domain.

Notch filters are used to remove selected frequencies from the Fourier spectrum in a specified neighborhood of a certain center frequency. This typology of filter has been used only for the input images in our work, since applying it to filter the strain field would require an a-priori knowledge of the expected strain distribution. Conversely notch filters are widely used in image processing where they can effectively improve image quality.

Once the coordinates of the centre of the area to be filtered ( and ) were selected, the following transfer function was applied:

$$
H(u, v)=1-e^{\frac{-1}{2}\left[\frac{D_{1}(u, v) D_{2}(u, v)}{D_{0}^{2}}\right](\text { Eq. 7) }}
$$

where:

- $\quad$ is the cutoff frequency of the Gaussian term;

- $\quad$ represents the distance from the centre of the spectrum of the points and;

The filtering of multiple frequencies can be performed by applying recursively the proper transfer functions, or adding together the Gaussian terms (one for each point ( and )) in a single transfer function. 


\subsection{COMBINING TECHNIQUES APPLIED TO THE IMAGES AND STRAIN DISTRIBUTIONS}

In this paper we analyze the effects of combining stacks of acquired images as well as stacks of strain distributions. An image combining strategy is based on the acquisition of a stack of images of the same scene. Combining a number of frames together reduces random noise and increases the signal-to-noise ratio [18]. The only requisite is that the scene must not change while the series of images is acquired. The various images acquired were combined through a pixel-by-pixel median. Unlike the mean, the median rejects abnormal pixels, without smoothing the image.

The same considerations can be applied to series of strain distributions. In this case instead of a pixel-by-pixel median combination, we computed a point-by-point median combination.

\section{PRELIMINARY ANALYSIS OF NOISE}

\subsection{DARK NOISE}

A preliminary analysis was conducted in order to determine the appearance and effects of dark noise in images acquired by the cameras of the DIC system. Covers were applied to the lenses in order to prevent light from entering the cameras, and the exposure time was set to the minimum allowed value: $0.1 \mathrm{~s}$. Using this procedure, the acquired image contained the noise generated by the electronics of the sensor and no 
actual information related to the light or heating of the CCD sensor. Six frames were collected at $0.5 \mathrm{~Hz}$ within one minute from turning the cameras on, so that the sensor would remain at room temperature. The six frames were then combined in order to calculate their pixel-by-pixel median. The result of this calculation is usually called "master bias frame" [9].

Subsequently, to perform measurements under the optimal conditions, the time needed by the cameras to stabilize their temperature was determined. Temperature measurements were taken every two minutes from the time when the cameras were switched-on, using a thermocouple placed on the camera metal casings, according to the manufacturer specifications (Figure 3a). After 50 minutes the temperature was stable at $39 \pm 0.5^{\circ} \mathrm{C}$ (Figure $3 \mathrm{~b}$ ). During this period of time the CCD sensors warmed up, and dark current had the time to produce its effects. Ten frames were recorded using different exposure times from 30 to $50 \mathrm{~ms}$. Blind covers were applied to the cameras. The images obtained contained both the noise generated by the electronics, and by the heating of the CCD sensor. Finally "thermal frames" were obtained subtracting the "master bias frame" from every frame recorded in the latter phase. Using this strategy, images containing dark noise generated by the heating of the sensor during the exposure time were generated.

The direct Fourier transform of the "thermal frame" showed a regular pattern in the frequency domain for all exposure times (Figure 4). 


\subsection{NOISE IN THE STRAIN DISTRIBUTIONS}

In this section we addressed the relationship between the strain magnitude, and the position and intensity of frequency peaks. The amount of noise and its characteristics were also analyzed, both in the strain distributions and in their Fourier spectra. The test was carried out by means of 15 loading steps in order to gather information on a large amount of strain levels. The tensile test was performed on the PTFE plate (Figure 2a), which was prepared with an optimized speckle pattern [32]. The specimen was first stretched in displacement control mode in 5 steps of $0.2 \mathrm{~mm}$ (each corresponding to an average strain increment in the order of $200 \times 10^{-6}$ at a strain rate of $50 \times 10^{-6}$ per second), followed by 10 steps of $1 \mathrm{~mm}$ (average strain in the order of $1000^{-} \times 10^{-6}$ at a strain rate of $250 \times 10^{-6}$ per second). To allow the viscoelastic time-dependent response to settle, a 50 -second interval was allowed between steps, and the DIC acquisitions were taken during the last 4 seconds of each interval. The specimen fractured around $8500 \times 10^{-6}$ thus exceeding the range $0-5000 \times 10^{-6}$ which was the focus of our study).

The strain distribution for each loading step was computed with reference to the initial unloaded state, thus enabling calculation of the strain (and of the noise) corresponding to 15 levels of strain. In order to focus on a constant-strain region, the calculation of the strain distribution via DIC was limited to the centre of the gage length $(50 \mathrm{~mm} \times 50 \mathrm{~mm})$. The strain distributions and their corresponding Fourier amplitude spectra were examined. 
As ideally a uniform strain distribution was expected in the analyzed constant-strain region, the uncertainty was calculated as the standard deviation among the DICcomputed strain data points. The uncertainty of the DIC-computed strain (i.e. the standard deviation of punctual strain values in the analyzed constant-strain region) did not change for the different levels of imposed strain (Table 3). Strain distributions having a low average magnitude were therefore most heavily corrupted by noise (Figure 5: a1 and b1). Conversely, noise affected to a lesser extent the strain distributions corresponding to high strain values. In fact the comparison of the Fourier amplitude spectra (Figure $5 \mathrm{a} 3$ and b3) shows both the constancy of high-frequency peaks, and the enhancement of low-frequency peaks associated with an increase of the average strain magnitude.

\section{FILTERING OF THE INPUT IMAGES}

\subsection{RATIONALE}

This part of the study aimed at assessing if a dedicated mild filtering on the acquired images (before they are fed into the DIC algorithm) can eliminate the noise component deriving from the image acquisition (which has some constant features, see above), without compromising the image sharpness, which is required for subsequent image correlation. 


\subsection{MATERIALS AND METHODS}

After calibrating the system for a $250 \mathrm{~mm} \times 200 \mathrm{~mm}$ field, ten images of the non-strained cylindrical aluminum bar were taken (Figure 2b). The calculation area was reduced to the rectangular surface under an angle of on the specimen, in order to exclude the less illuminated zones. The apparent strain distributions computed by the software were compared to the theoretical strain (equal to zero) by means of the root mean square error (RMSE Eq. 2).

In this experiment the acquired images were exported from the ARAMIS software, and fed into custom-written Matlab programs to test two different filtering strategies:

- The first approach consisted in the application of a median adaptive filter. Convolution kernels of dimensions variable from $1 \times 3$ pixels to $3 \times 3$ pixels were tested.

- A second approach was based on a spatial frequency domain filtering strategy. The Fourier amplitude spectrum was computed for the previously acquired images. As the ten images were acquired consecutively under constant strain conditions, their Fourier amplitude spectra were theoretically identical. The Fourier spectrum of each acquired image was subtracted from the corresponding reference image, and a logarithmic transformation of the intensity values was computed to enhance the higher frequency components (Figure 6). The comparison in the frequency domain showed regularity in the positioning of 
some peaks. The analysis of the effects of dark currents in the acquired images previously conducted (Figure 3 ) confirmed that these peaks were caused by dark noise. To remove the corresponding frequencies, Gaussian Notch filters were used. In particular six series of Gaussian Notch reject filters centered in the 12 higher intensity noise frequencies were implemented. The series differed one from the other with regard to the cutoff frequency, which varied from 1 to 100 pixels. This generated different series of filtered images.

The filtered images were reintroduced in the ARAMIS DIC software and reprocessed to compute the strain distribution. To compare the results, the RMSE values from the theoretical strain value equal to zero were computed.

\subsection{RESULTS}

Compared to the strains derived from the originally captured images, noise increased when strains were computed from images filtered using a $3 \times 1$ kernel; such deterioration was even more pronounced for the $3 \times 3$ kernel (Figure $7 \mathrm{a}$ ).

On the other hand the RMSE values did not seem to be affected by the application of Notch filters until the cutoff frequency was less than twelve pixels (Figure 7b). For a cutoff frequency of 50 pixels the noise increased significantly. A cutoff frequency of 100 pixels caused a complete loss of correlation. 


\section{FILTERING THE STRAIN DISTRIBUTIONS IN THE SPATIAL FREQUENCY DOMAIN}

\subsection{RATIONALE}

As filtering the input images was not an effective strategy, this part of the study focused on filtering the DIC-computed strain distributions and finding an optimum in the application of filters on the spatial frequency domain. The optimal cutoff frequency was searched in order to discriminate between the actual noise (to be removed as much as possible), and real strain gradients (to be preserved as much as possible).

\subsection{STRAIN DISTRIBUTIONS AND THEIR FOURIER SPECTRA}

The first step to build a filtering strategy in the spatial frequency domain was the understanding of the position and intensity of the peaks in the Fourier spectra of the strain distributions. To achieve this result, the cantilever test structure was used (Figure 2c). The system was calibrated for a $250 \mathrm{~mm} \times 200 \mathrm{~mm}$ field. The aluminum bar was initially observed in the non-strained conditions. Strains were computed in a region of $270 \mathrm{~mm} \times 150 \mathrm{~mm}$. The longitudinal $(\mathrm{x})$ and transversal $(\mathrm{y})$ components of strain were computed by the DIC software. The corresponding Fourier amplitude spectra were extracted. In a second phase the bar was observed when a $5 \mathrm{~mm}$ deflection was imposed at the free end (corresponding to a maximum strain of $300 \times 10^{-6}$ ). 
The Fourier amplitude spectra of the strain distributions obtained using the cantilever test structure were compared. It was possible to notice some regularity in position and directionality of the peaks (Figure 8). The main difference between spectra for the longitudinal (x) and transversal (y) strain components was the directionality of the peaks: a $90^{\circ}$ shift was recognizable. The main difference between the spectrum of the non-strained aluminum plate, and spectrum of the strained one was the enhancement of the central peaks. The high-frequency peaks (which correspond to noise) remained unaffected by application of a physical strain to the specimen.

\subsection{IMPLEMENTATION OF THE FILTER}

A filtering strategy in the frequency domain was implemented. Results of Section 3 suggest using a Gaussian low-pass filter in order to:

1. Preserve the low-frequency components, which contain information associated with existing strain gradients.

2. Remove the high-frequency peaks, which are associated with noise.

3. Enable tuning the cutoff frequency to optimize the removal of noise while minimizing the associated loss of information (smoothing of existing strain gradients).

Starting from the results of the test on the PTFE plate (Section 3.2) it is possible to observe that the peaks corresponding to noise remain constant in position and intensity 
independently of the magnitude of the applied strain. Using this feature, the Fourier spectrum of the apparent strain generated by noise can be used for every observation as a tool to describe noise in the frequency domain.

Using the cantilever test structure (Figure 2c), a series of 22 observations was created. The aluminum plate underwent increasing deflections at the free end up to $13 \mathrm{~mm}$ (corresponding to a maximum strain value at the fixed end of $750 \times 10^{-6}$ ). Displacement and strain was measured synchronously in two independent ways:

- The strain distribution for each observation was computed using the DIC system.

- At the same time, seven LVDTs placed at different distances from the fixed end of the plate were used to accurately measure the deflection (Figure 2c-d). The strain distribution of the plate could then be accurately computed: the LVDTmeasured displacements were fitted using the beam equation for a bending moment, including an elastic constraint of the fixed end of the aluminum plate.

- DIC-computed strains could thus be compared against the LVDT-based measurements, which were assumed as a reference.

The frequency representations of the actual strain distribution (information) and of the noise were separately available for each of the 22 strain distributions acquired. The superimposition principle was applied to evaluate separately the action of the filter in terms of noise removal, and loss of information. 
The procedure to choose the optimal cutoff frequency for the low-pass filter followed these steps:

1. The apparent strain was filtered using a series of Gaussian low-pass filters of different cutoff frequencies. Using the notation described in (Eq. 6), the value $D_{0}$ of in the series of implemented filters, varied from 0 to 10 in 0.1 increments.

2. The amount of noise not removed by the low-pass filter was estimated by computing the RMSE of the filtered DIC-computed apparent strain in the unstrained condition:

$$
\text { ResidualNoise }=R M S E_{k}=\sqrt{\frac{1}{n} \frac{1}{m} \sum_{i=1}^{n} \sum_{j=1}^{m}\left(\varepsilon_{D I C+\text { filter }, i, j}^{2}-0\right)} \text { (Eq. 8) }
$$

where:

- $k$ is the cutoff frequency implemented in the current filter analyzed;

- $\quad n \times m$ are the number of points composing the calculation area;

- $\quad$ is the filtered DIC-computed apparent strain at each point;

- 0 is the theoretical strain value in the unstrained condition.

The RMSE here is an indicator of the noise not removed (RMSE would be zero if noise was totally removed). 
3. The same series of filters applied at point 1 . was applied to the theoretical strain distributions derived from the LVDT measurements.

4. We evaluated the amount of information removed by filters with different cutoff frequencies computing the RMSE between the non-filtered and filtered theoretical distributions:

Loss of information $=R M S E_{s, k}=\sqrt{\frac{1}{n} \frac{1}{m} \sum_{i=1}^{n} \sum_{j=1}^{m}\left(\varepsilon_{L V D T+f i t e r, i, j}-\hat{\varepsilon}_{L V D T, i, j}\right)^{2}}$

where:

- $\quad s$ is the acquisition considered;

- $\quad k$ is the cutoff frequency implemented in the current filter analyzed;

- $\hat{\varepsilon}_{L V D T, i, j}$ is the theoretical strain value at each point (derived from the LVDT measurements).

- $\varepsilon_{L V D T+f i t e r, i, j}$ is the strain value obtained ad at each point after filtering the theoretical strain distribution.

5. The total (quadratic) error was calculated for each observation and for each cutoff frequency, using the values from Eq. 8-9:

$$
\text { TotalError }=\text { Quadratic Sum }=T_{s, k}=\sqrt{\left(R M S E_{k}\right)^{2}+\left(R M S E_{s, k}\right)^{2}} \text { (Eq.10) }
$$

\subsection{RESULTS}


A decrease of the residual noise was detectable when the cutoff frequency was increased (Figure 9). At the same time the loss of information increased when a higher cutoff frequency was chosen. The quadratic sum had a minimum: the corresponding cutoff frequency is the one to be chosen to optimize the filtering process. On the other hand, applying the filter without any optimization criteria can compromise the results amplifying the loss of information. The optimal cutoff frequency was identified for each level of applied strain (Table 4). There is no theoretical way of predicting such an optimal value a priori. Such an optimum depended on the spatial subdivision applied when processing the images acquired (i.e. number of samples), but also on the level of applied strain. The selected Gaussian low-pass filtering effectively reduced the noise affecting the strain distribution, while preserving in the most reliable way the existing strain gradient (that is the real information) (Figure 10). Some differences could still be detected between the theoretical distribution and the filtered one (Figure 10c and 10d). There are two concurring reasons for strain map not being perfectly symmetrical: (i) unavoidable experimental error (e.g. boundary conditions differing from an ideal constraint; slightly off-axis force; slightly irregular shape of the cantilever bar, etc); (ii) DIC errors (both systematic and random). Such errors combine through the measurement chain so that they cannot be separated in the DIC-computed strain distribution. It is also important to stress that the optimal filtering radius cannot be determined a-priori, since the shape and dimension of the frequency peaks are highly influenced by the characteristics of the distribution of the calculation points. This implies that if a certain frequency carries information for a certain combination of 
calculation points, it may carry just noise for another. It must be clear that there is no fit-for-all solution, as the optimal cutoff frequency depends on the noise (i.e. on the DIC hardware and software), and on the strain gradients (i.e. on the mechanical problem under investigation). Therefore, the optimal cutoff frequency needs to be assessed for each experiment, based on the noise actually present in the DIC-computed strain. 


\section{COMBINATION TECHNIQUES APPLIED TO THE ACQUIRED IMAGES AND TO THE STRAIN DISTRIBUTIONS}

\subsection{RATIONALE}

This part of the study focused on the time-dependent component of noise (i.e. noise deriving from air turbulence, vibrations, illumination etc.). These phenomena cause randomly distributed noise in the images, and are usually referred to as Gaussian noise sources [18]. They create uniform and equally-distributed peaks in the Fourier spectrum. Two strategies were explored that are potentially effective in removing such components of noise.

\subsection{MATERIALS AND METHODS}

The last filtering strategy explored was the combination of stacks of images: this was applied both to the acquired images, and to the strain distributions. This approach is built on multiple acquisitions of the same event. The acquisitions are then combined in order to reduce random components of noise, either in the input images, or in the DICcomputed strain distributions.

The experiment was carried out using the cantilever test structure where the LVDT were placed as in the previous experiments (Figure $2 \mathrm{c}-\mathrm{d}$ ). The DIC was calibrated for a measuring window of $250 \mathrm{~mm} \times 200 \mathrm{~mm}$. Images of the aluminum plate were acquired without a load, and for twelve steps of increased deflection at the free end $(1 \mathrm{~mm}$ per step). For each step ( 1 unloaded +12 loaded), 20 theoretically identical images were 
recorded using a $15 \mathrm{~Hz}$ frame rate. The strain distributions were on the whole surface of the specimen. For each step and each image, the image itself and the DIC-computed strain distribution were exported. Furthermore, the theoretical strain distribution was computed on the basis of the LVDT measured deflections. As a baseline, the noise of the unfiltered DIC-computed distribution was evaluated as RMSE compared to the theoretical strain distribution, creating a series of 260 RMSE values (20 for each of the $(1+12)$ steps $)$.

The exported images and strain distributions were then elaborated using two different procedures:

1. A pixel-by-pixel median of the theoretically identical 20 images groups was created. A stack of 13 "median images" was created using this method corresponding to the 13 load steps. The 13 "median images" were reintroduced into the DIC software and processed to compute the strain distribution. The RMSE between the strain distributions obtained using the "median images" and the theoretical one (derived from the LVDT measurements) were computed.

2. A point-by-point median of the theoretically identical 20 DIC-computed strain distributions groups was created. In this way, 13 "median strain distributions" were available. Their 13 RMSE values from the theoretical strain distribution (derived from the LVDT measurements) were computed. 


\subsection{RESULTS}

An average $10 \%$ decrease in terms of RMSE was noticeable after the creation of the "median images" (Figure 11). The creation of "median strain distributions" did not significantly reduce the error.

\section{DISCUSSION}

The aim of this study was to systematically explore filtering strategies to reduce noise in strain measurements using DIC, and to quantify the noise reduction that can be achieved, together with the corresponding loss of information obtained. Strategies involving both the input images and the computed strain distributions were explored.

To some extent, the present findings are specific to the Aramis system. However, as (i) this is one of the most common commercial systems, and (ii) most commercial systems deploy similar algorithms, most observations can be extended to other equivalent DIC systems.

At first we focused on the spatial and frequency domain appearance of noise in the strain distributions for a typical strain range $\left(0-5000 \times 10^{-6}\right)$. Noise was not negligible (it easily exceeded $\left.160 \times 10^{-6}\right)$, and could hide the real strain distribution. However, noise had constant RMSE amplitude, independent of the strain magnitude it was superimposed to. This feature is confirmed by the appearance of Fourier spectra in this range of strain values: high-frequency peaks (corresponding to noise) are constant in 
position and intensity, while the low-frequency ones (corresponding to real strain gradients) increase their intensity with increased of stress levels.

Filtering the input images led to a detrimental loss of contrast and increased noise in the computed strain distributions, even using an adaptive implementation. Removing thermal noise in the input images by subtracting a "thermal frame" produced very limited noise reduction in the computed strain. On the other hand an extensive use of Notch reject filters damaged the input images and led to de-correlation.

Filters involving Fourier spectra of strain distributions were then evaluated. A Gaussian low-pass filter was implemented in order to remove high-frequency peaks and a recursive procedure to calculate the optimal cutoff frequency of the filter was created to minimize the total error (i.e. the sum of the residual noise and of the loss of information). This feature indicated that information was expressed by a reduced number of frequencies in the Fourier spectrum. When the cutoff frequency of the lowpass filter was optimal, the residual noise and the loss of information generated were of the same order of magnitude. The Gaussian low-pass filter was able to reduce the error by up to $70 \%$, when the optimal cutoff frequency was chosen. This left a residual noise of the order of $20 \times 10^{-6}$ (RMSE), when the original strain distribution was affected by a noise of $160 \times 10^{-6}(\mathrm{RMSE})$, while the total error was of $90 \times 10^{-6}$. This study showed the importance of the optimization process when applying the low-pass filtering: a nonoptimal cutoff frequency can corrupt the strain distribution causing high loss of information. Unfortunately the cutoff frequency cannot be determined a-priori, but 
must be assessed for each experiment, based on the noise observed in the DICcomputed strain distribution.

Finally, image combination techniques were explored. Application of the combination technique to the acquired images produced a limited reduction $(10 \%)$ of the noise in the computed strain distributions. Application of the combining technique to the computed strain distributions did not lead to any significant benefit. While image combination provided very limited benefit and it was very time-consuming.

There are few studies in the literature addressing the filtering strategies involving strain distributions filters. The results from our study confirmed the reasons of the interest that frequency domain filters have recently gained [15].

A first limitation of this work is that we did not consider any strategy involving the filtering of displacement distributions, and their reintroduction in the DIC software in order to calculate strain distributions. Unfortunately the closed nature of most commercially available DIC software makes this strategy impracticable.

Although the low-pass spatial frequency domain filtering seems most promising, there are some limitations that must be addressed. In fact, the whole process to calculate the optimal cutoff frequency requires an estimate of the strain gradients. This is feasible only if simple stress configurations and linear materials are considered. If these conditions are not satisfied, the filtering approach can proceed only with qualitative bases. 
This study was carried out when the DIC was calibrated for a $250 \mathrm{~mm} \times 200 \mathrm{~mm}$ measurement window. The scale factor was not considered in the experimental activity and only one size of pattern was characterized. However, this does not limit the generality of this study. In fact, scalability of the results is guaranteed once the pattern on the specimen meets some theoretical requirements (i.e. when the average size corresponds to at least three pixels $[3,7,32,34])$.

\section{CONCLUSIONS}

Several filtering techniques were explored to reduce noise in DIC-computed strains. Filtering techniques involving input images and median image combining revealed to be inefficient or led to an increase of noise in the strain distributions. Gaussian low-pass filtering to remove noise from the DIC-computed strain distributions was the only truly successfully strategy. This strategy resulted in a noise reduction up to $70 \%$, without significant loss of information when the filter settings were optimized. Such an optimization is applicable only when some information is available a-priori about the strain gradients (e.g. for simple geometries, and/or when studying linear-elastic Hookean materials). One should be aware that filtering must always be performed with caution, and with a deep understanding of the side-effects of filtering. In fact, if filtering is applied without considering the existing strain gradients (i.e. only focusing on the reduction of the visible noise), this can results in serious loss of information, whereas errors can be significantly reduced when the filter is properly selected. 


\section{Conflict of interest statement}

There is no potential conflict of interest: none of the Authors received or will receive direct or indirect benefits from third parties for the performance of this study. 


\section{REFERENCES}

1. Freddi, A., Olmi, G. \& Cristofolini, L. (2015) Experimental Stress Analysis for Materials and Structures. 4, Springer International Publishing. doi: 10.1007/978-3-319-06086-6

2. Tong, W. (2005) An Evaluation of Digital Image Correlation Criteria for Strain Mapping Applications. Strain, 41(4), 167-175. doi:10.1111/j.1475-1305.2005.00227.x

3. Sutton, M. A., Orteu, J.-J., \& Schreier, H.W. (2009) Image correlation for shape, motion and deformation measurements: basic concepts, theory and applications. Springer.

4. Choi, S., \& Shah, S.P. (1997). Measurement of deformations on concrete subjected to compression using image correlation. Experimental Mechanics, 37(3), 307-313. doi:10.1007/BF02317423

5. Amiot, F., Bornert, M., Doumalin, P., Dupré, J.-C., Fazzini, M., Orteu, J.-J., Wienin, J.S. (2013) Assessment of Digital Image Correlation Measurement Accuracy in the Ultimate Error Regime: Main Results of a Collaborative Benchmark: Benchmark on Digital Image Correlation Ultimate Error. Strain, 49(6), 483-496. doi:10.1111/str.12054

6. Proakis, J. G., \& Manolakis, D. G. (2006) Digital Signal Processing - Principal, Algorithms and Applications (4th ed.). Pearson Prentice Hall.

7. Le Cam, J.-B. (2012) A Review of the Challenges and Limitations of Full-Field Measurements Applied to Large Heterogeneous Deformations of Rubbers: Review of the Challenges and Limitations of Full-Field Measurements. Strain, 48(2), 174-188. doi:10.1111/j.14751305.2011.00830.x

8. Wang, Y., Lava, P., Coppieters, S., De Strycker, M., Van Houtte, P., \& Debruyne, D. (2012) Investigation of the Uncertainty of DIC Under Heterogeneous Strain States with Numerical Tests: 2D DIC under Heterogeneous Strain State. Strain, 48(6), 453-462. doi:10.1111/j.14751305.2012.00840.x

9. Janesick, J. R. (2001) Scientific Charge-coupled Devices. SPIE Press.

10. Stuart, A. M. (2006) CCD Astrophotography: High-Quality Imaging from the Suburbs: HighQuality Imaging from the Suburbs. Springer.

11. Correlated Solutions (Ed.). (2010) Vic-3D 2010, Reference Manual. Retrieved from http://www.correlatedsolutions.com/index.php/downloads on $<$ December 2013> 
12. Fung, Y.C. in Biomechanics 383-415 (Springer New York, 1981) doi: 10.1007/978-1-4757-1752$5 \_12$

13. Zhou, Y., Sun C., Song Y. , Chen J (2014) Image pre-filtering for measurement error reduction in digital image correlation, Opt. Laser. Eng. 01/2015; 65. doi: 10.1016/j.optlaseng.2014.04.018.

14. Mazzoleni, P., Matta, F., Zappa E., Sutton M., Cigada A. (2015) Gaussian pre-filtering for uncertainty minimization in digital image correlation using numerically-designed speckle patterns, Opt. Laser. Eng. 66(2015)19-33

15. Mortazavi, F., Lévesque, M., \& Villemure, I. (2013) Image-based Continuous Displacement Measurements Using an Improved Spectral Approach: Image-based Continuous Displacement Measurements. Strain, 49(3), 233-248. doi:10.1111/str.12031

16. GOM mbH, Braunschweig, Germany. (2007). ARAMIS, v 6.0 User's Manual.

17. Dantec Dynamics GmbH. (2012) Istra 4d DIC Software manual - Elektronic (2.4.0 ed.).

18. Gonzalez, R.C. (2009) Digital Image Processing. Pearson Education.

19. Hu, Z., Xie, H., Lu, J., Hua, T., \& Zhu, J. (2010) Study of the performance of different subpixel image correlation methods in 3D digital image correlation. Applied Optics, 49(21), 4044-4051. doi:10.1364/AO.49.004044

20. Schreier, H.W., \& Sutton, M.A. (2002) Systematic errors in digital image correlation due to undermatched subset shape functions. Experimental Mechanics, 42(3), 303-310. doi:10.1007/BF02410987

21. Luu, L., Wang, Z., Vo, M., Hoang, T., \& Ma, J. (2011) Accuracy enhancement of digital image correlation with B-spline interpolation. Optics Letters, 36(16), 3070-3072. doi:10.1364/OL.36.003070

22. Barranger, Y., Doumalin, P., Dupré, J.C., \& Germaneau, A. (2012) Strain Measurement by Digital Image Correlation: Influence of Two Types of Speckle Patterns Made from Rigid or Deformable Marks: Strain Measurement by Digital Image Correlation. Strain, 48(5), 357-365. doi:10.1111/j.1475-1305.2011.00831.x

23. Hild, F. \& Roux, S. (2012), Comparison of local and global approaches to digital image correlation, Experimental Mechanics, 52 (9), 1503-1519. 
24. Besnard, G., Hild, F.\& Roux, S. "Finite-Element" displacement fields analysis from digital images: application to Portevin-Le Châtelier bands, Exp Mech, 46 (6) (2006), pp. 789803 http://dx.doi.org/10.1007/s11340-006-9824-8

25. Pierré, J.-E. , Passieux, J.-C. , Périé, J.-N. , Bugarin, F. \& Robert, L. Unstructured finite elementbased digital image correlation with enhanced management of quadrature and lens distortions, Optics and Lasers in Engineering, Volume 77, february 2016, Pages 44-53, ISSN 01438166,http://dx.doi.org/10.1016/j.optlaseng.2015.07.008.

26. Réthoré, J. ,. Elguedj, T, Simon, P. \& Coret, M. On the Use of NURBS functions for displacement derivatives measurement by digital image correlation Exp Mech, 50 (7) (2010), pp. 10991116 http://dx.doi.org/10.1007/s11340-009-9304-z

27 Avril, S., Feissel, P., Pierron, F. \& Villon, P., (2010), Comparison of two approaches for differentiating full-field data in solid mechanics, Measurement Science and Technology, 21 (1), doi:10.1088/0957-0233/21/1/015703

28. Lava, P., Cooreman, S. Coppieters, M. De Strycker, \& D. Debruyne. (2009) Assessment of Measuring Errors in DIC Using Deformation Fields Generated by Plastic FEA. Optics and Lasers in Engineering, 47(7-8), 747-53. doi:10.1016/j.optlaseng.2009.03.00724. Doumalin, P., Dupré, J. C., \& Germaneau, A. (2010). Digital Image Correlation accuracy: influence of kind of speckle and recording setup. EPJ Web of Conferences, 6, 31002. doi:10.1051/epjconf/20100631002

29. Yaofeng, S., \& Pang, J.H.L. (2007) Study of optimal subset size in digital image correlation of speckle pattern images. Optics and Lasers in Engineering, 45(9), 967-974. doi:10.1016/j.optlaseng.2007.01.012

30. Callister, W.D. (2007) Materials science and engineering: an introduction. New York: John Wiley \& Sons.

31. Teflon ${ }^{\circledR}$ PTFE Technical Data Sheets.

at: $<$ http://www.matweb.com/search/GetMatlsByTradename.aspx?navletter=T\&tn=Teflon\%C2\%A E+PTFE $>$

32. Lionello, G., Cristofolini L. (2014) A practical approach to optimizing the preparation of speckle patterns for digital-image correlation. 2014 Meas. Sci. Technol. 25, 107001. doi:10.1088/0957- 
0233/25/10/10700128. Covington, M. A. (2007). Digital SLR Astrophotography. Cambridge University Press.

33 Bertero M., Boccacci P. (1998) Introduction to Inverse Problems in Imaging, IoP

34. Hua, T., Xie, H., Wang, S., Hu, Z., Chen, P., \& Zhang, Q. (2011) Evaluation of the quality of a speckle pattern in the digital image correlation method by mean subset fluctuation. Optics \& Laser Technology, 43(1), 9-13. doi:10.1016/j.optlastec.2010.04.010 


\section{CAPTIONS TO FIGURES}

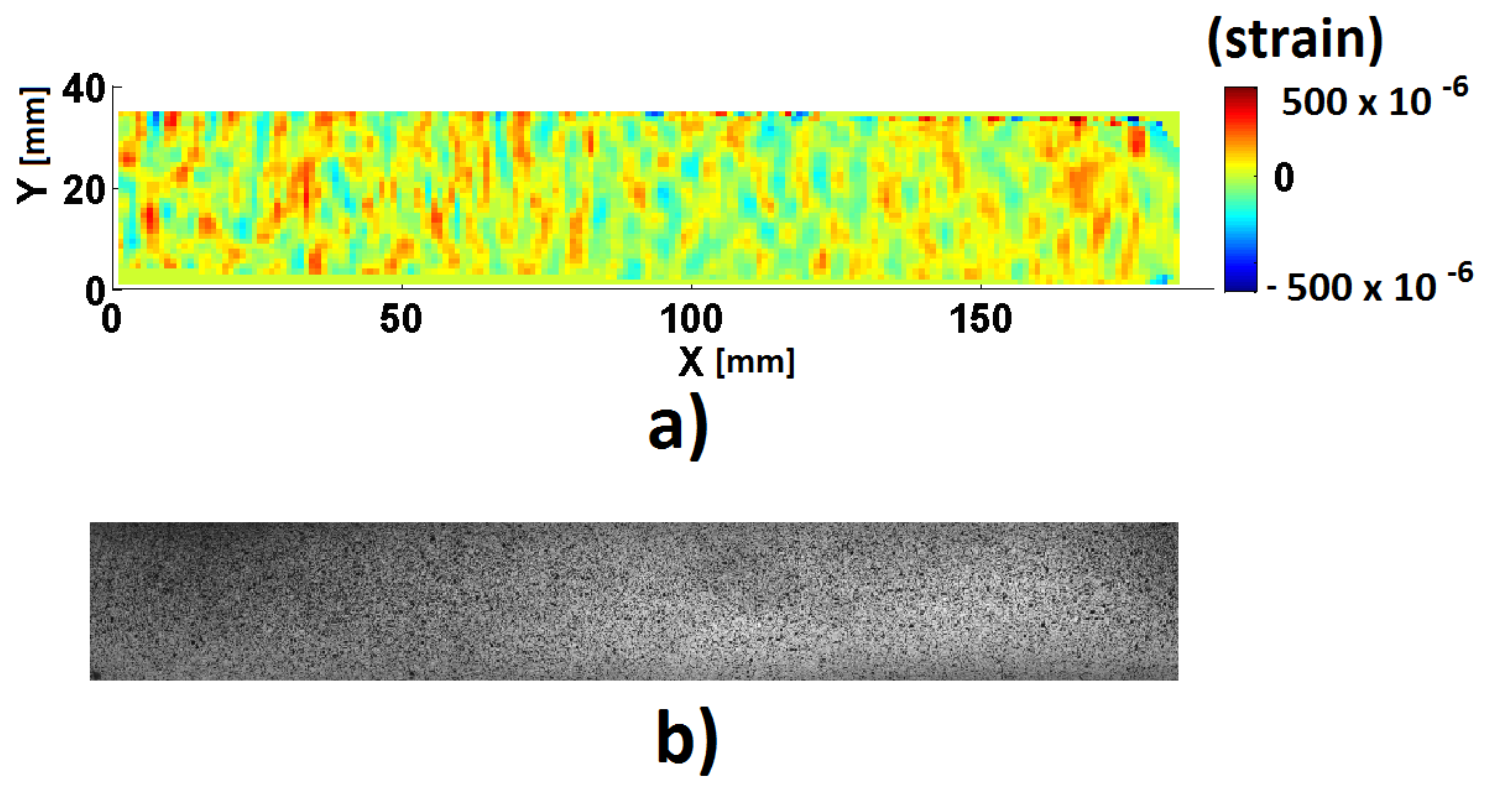

Figure 1 - Sub-figure a) shows the typical noise affecting strain measurement with DIC: while the object is not subjected to any physical strain, a remarkable noise pattern appears in the unfiltered DIC-computed strain distribution. Sub-figure b) shows the speckle pattern of the specimen. Speckles had an average dimension of 2.65 pixels with a 1.80 standard deviation. 


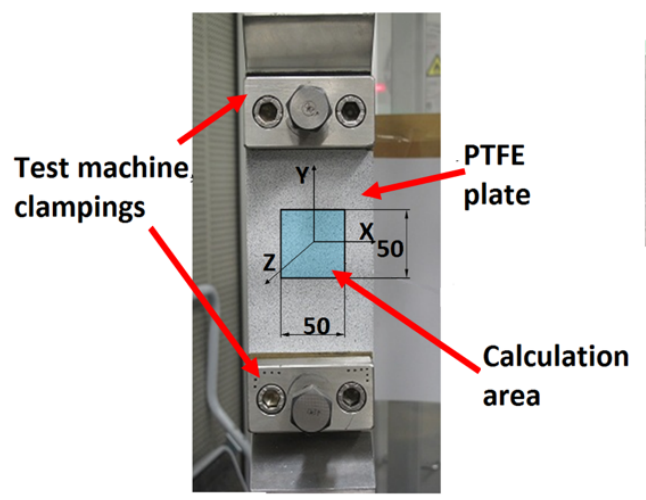

a)

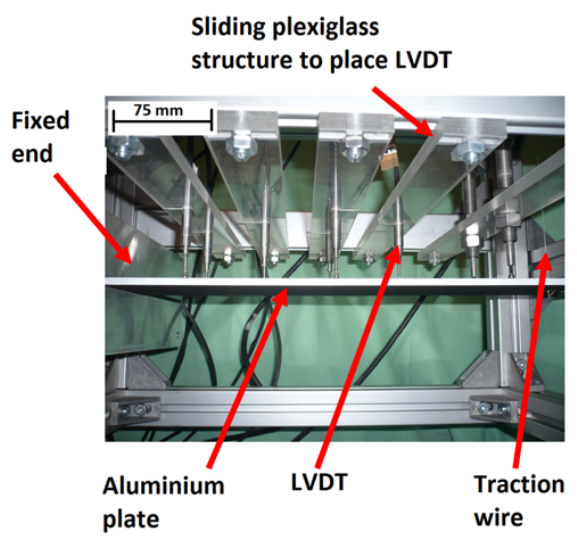

d)
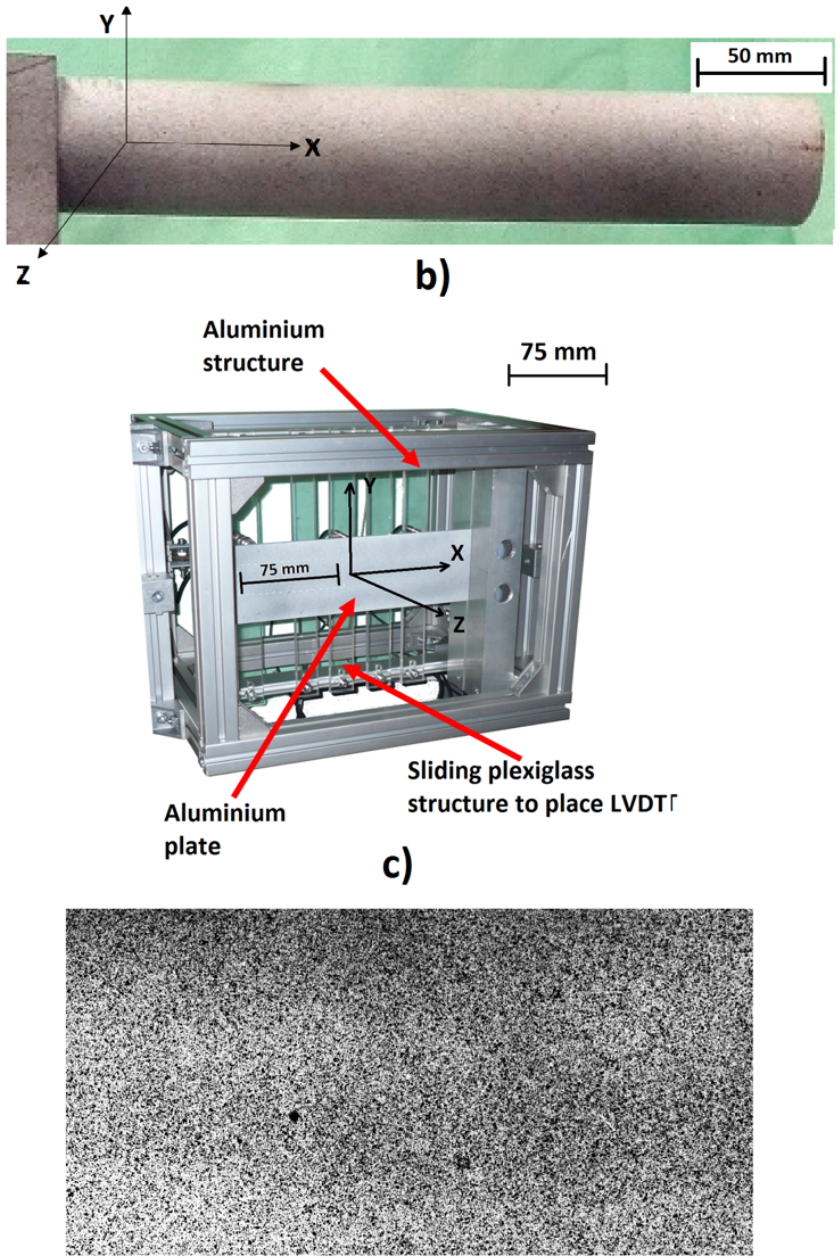

e)

Figure 2 - Instrumentation used in the experiments. Sub-figure a) shows the PTFE plate used in the tensile test. The blue square region corresponds to the $50 \mathrm{~mm} \times 50 \mathrm{~mm}$ area used in the DIC calculation. Sub-figure b) shows the aluminium cylindrical bar used for the digital image filtering process exploration. Sub-figure c) shows the cantilever aluminium structure frontally. Sub-figure d) shows the aluminium cantilever structure from above: the traction wire to create the deflection is visible, together with the LVDT and the sliding Plexiglass supports. Sub-figure e) shows the pattern on the aluminum cantilever structure. Mean speckle dimension was 2.0 pixels, with a standard deviation of 1.3. The facet size used for the elaboration of this pattern was 10 pixels [32] 

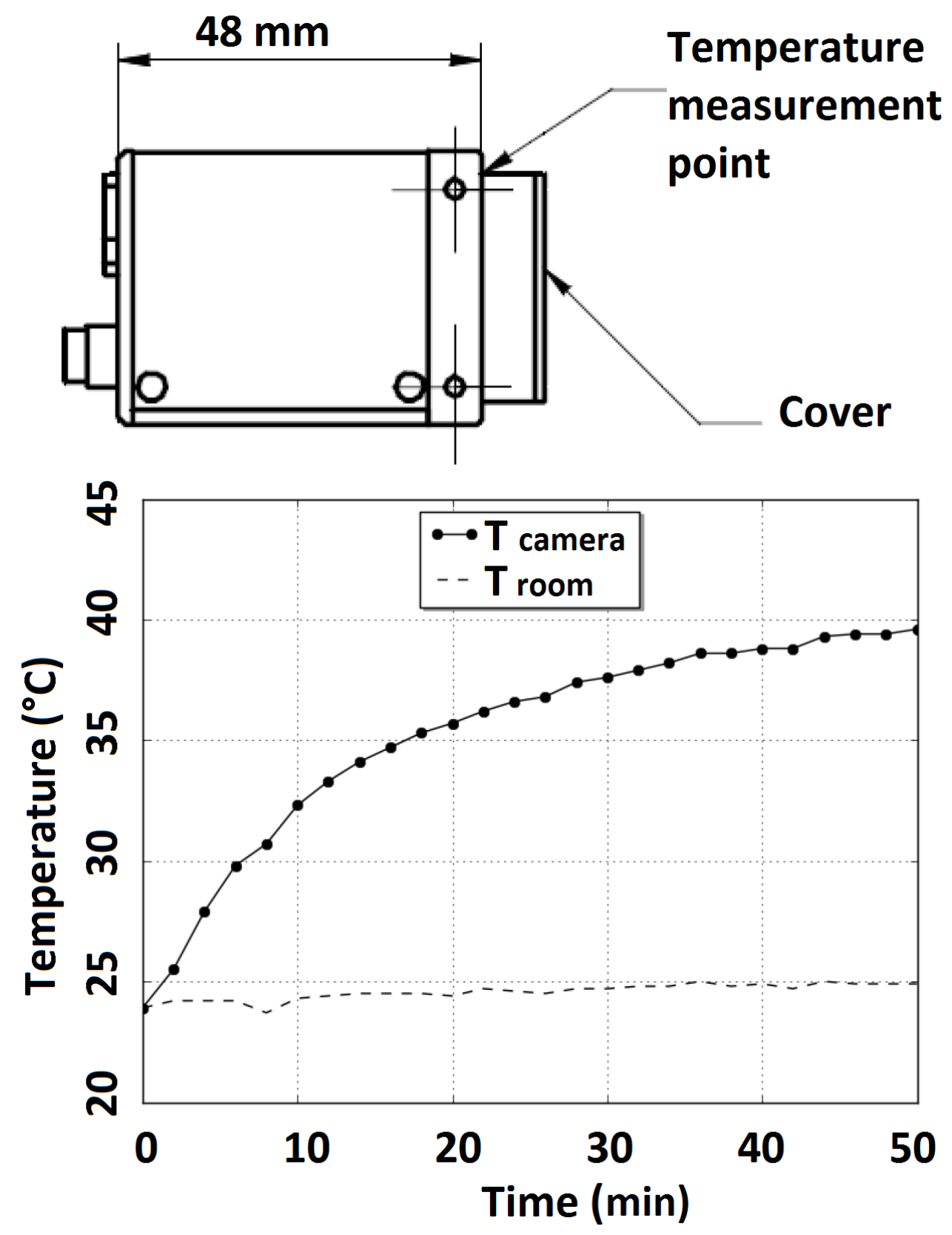

Figure 3 - Setup and results of the cameras temperature determination experiment. (a) A view of the camera from above, showing the point where the thermocouple was placed. (b) Variation of camera and room temperature over time: in 50 minutes the temperature of the CCD camera raised from the initial room temperature to the final $39^{\circ}$ C. The test was stopped when the difference between five consecutive temperature recordings was below $0.5^{\circ} \mathrm{C}$. 


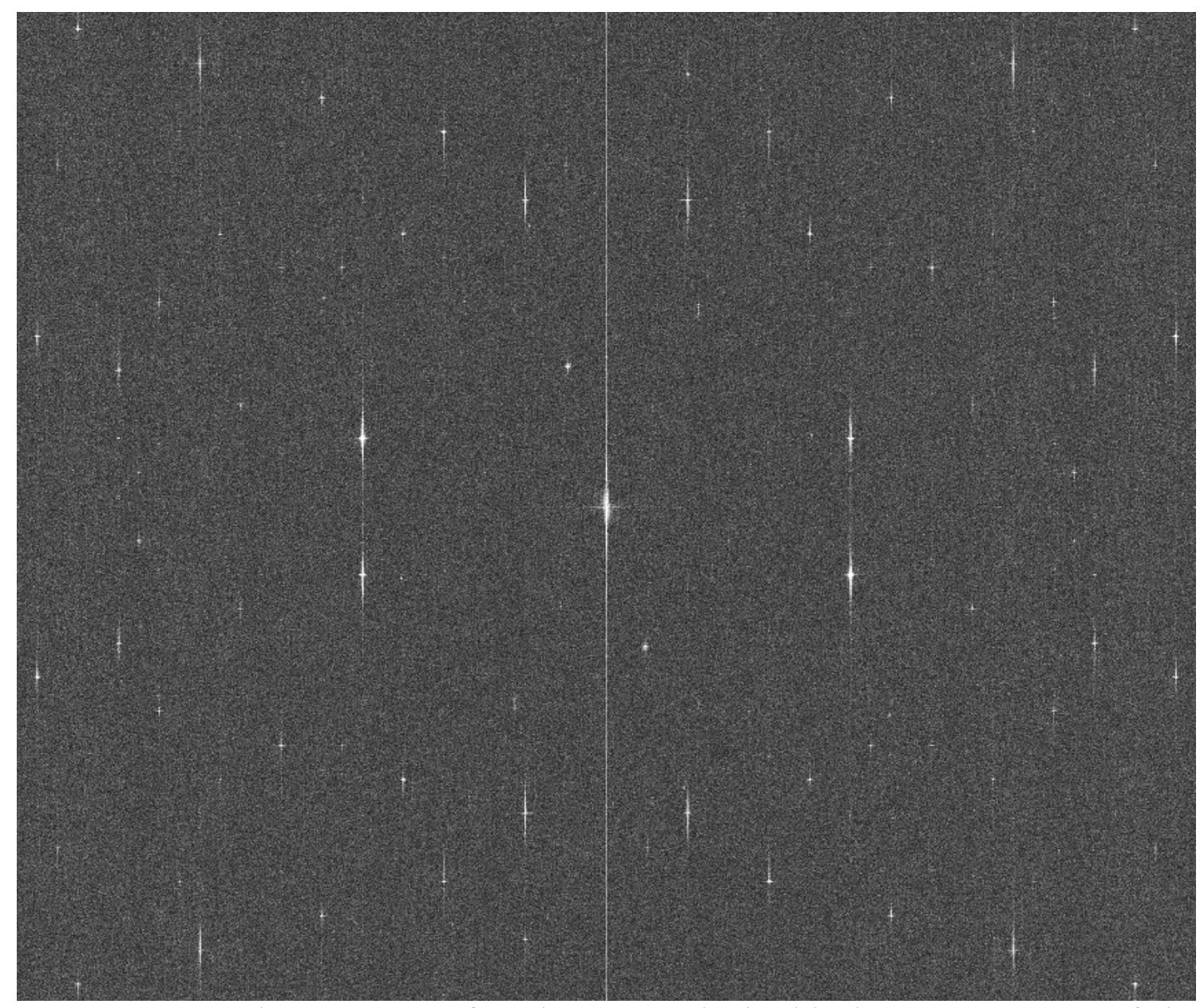

Figure 4 - Fourier spectrum of an image acquired with the CCD cameras of the ARAMIS system totally obscured. The white spots are the noise frequencies generated by dark noise in the "thermal frame". This noise comes from the surface of the CCD sensor: here an electron can be excited into the conduction band due to thermal reasons, resulting in random artifacts. The noise frequencies were underlined using an appropriate grey-level enhancement transformation. Similar results were obtained with all exposure times. 

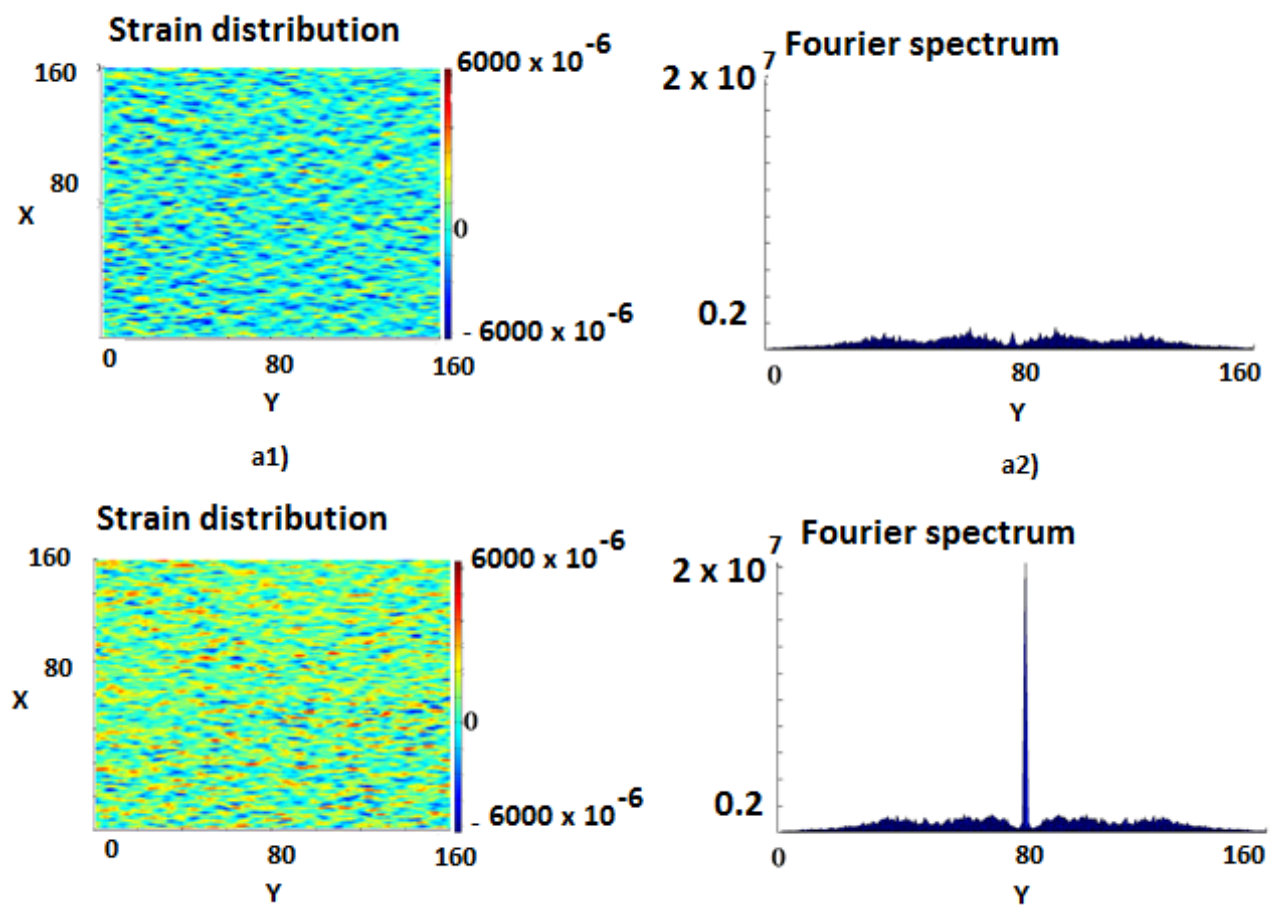

a1)

a2)
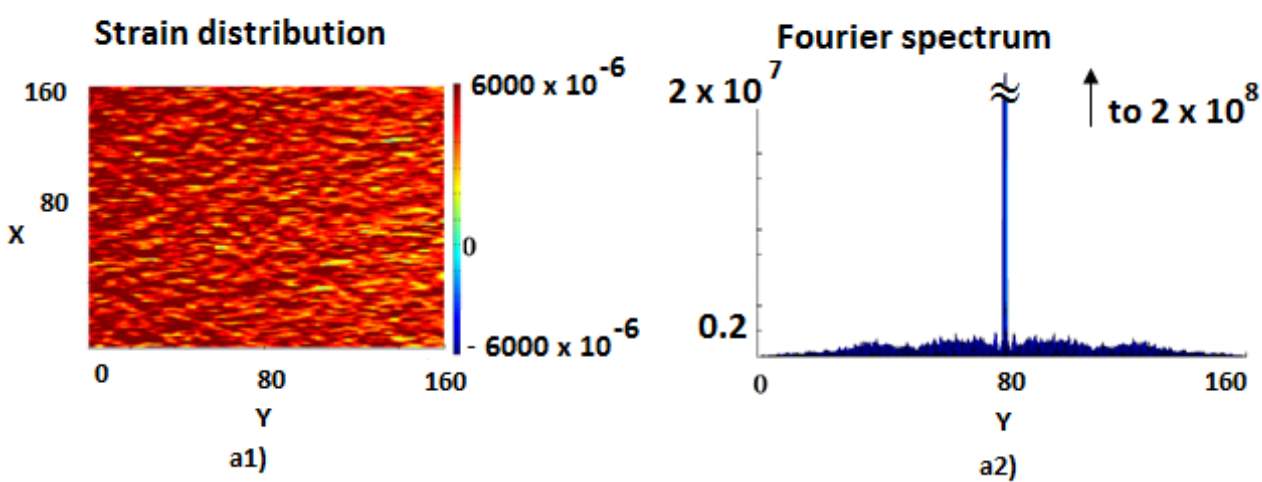

Figure 5 - Results of the tensile test for three of the total 15 loading steps analyzed on the PTFE plate. Sub-figures a1) and a2) are related to the unloaded specimen and show respectively the distribution of the $\mathrm{y}$ - strain component and the projection along the $\mathrm{y}-\mathrm{z}$ plane of the Fourier amplitude spectrum representation. Sub-figures b1) and b2) show the same quantities for an average strain values of approximately $500 \times 10^{-6}$ while $\mathrm{c} 1$ ) and c2) refer to an average strain value of $5000 \times 10^{-6}$. These three loading steps were chosen as most representative in order to give a meaningful description of the whole range of results obtained. A slight anisotropy of the strain noise can be observed: this is probably related to the uni-axial tensional distribution induced in the test specimen. 

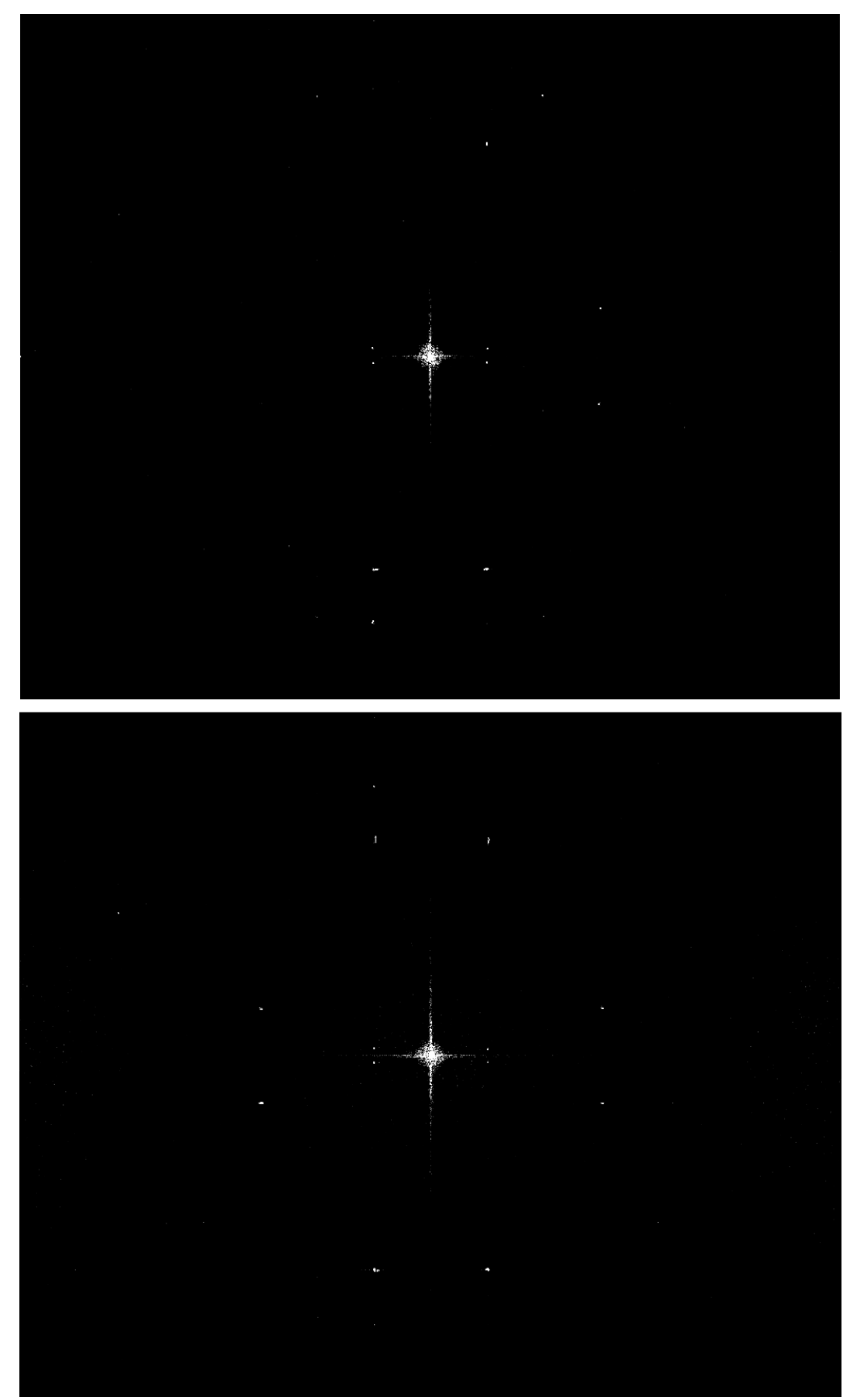

Figure 6 - Two examples of Fourier spectra obtained subtracting the amplitude spectrum of the reference image from the first (top) and the tenth (bottom) acquired image. The white points represent the position of higher intensity frequencies. 

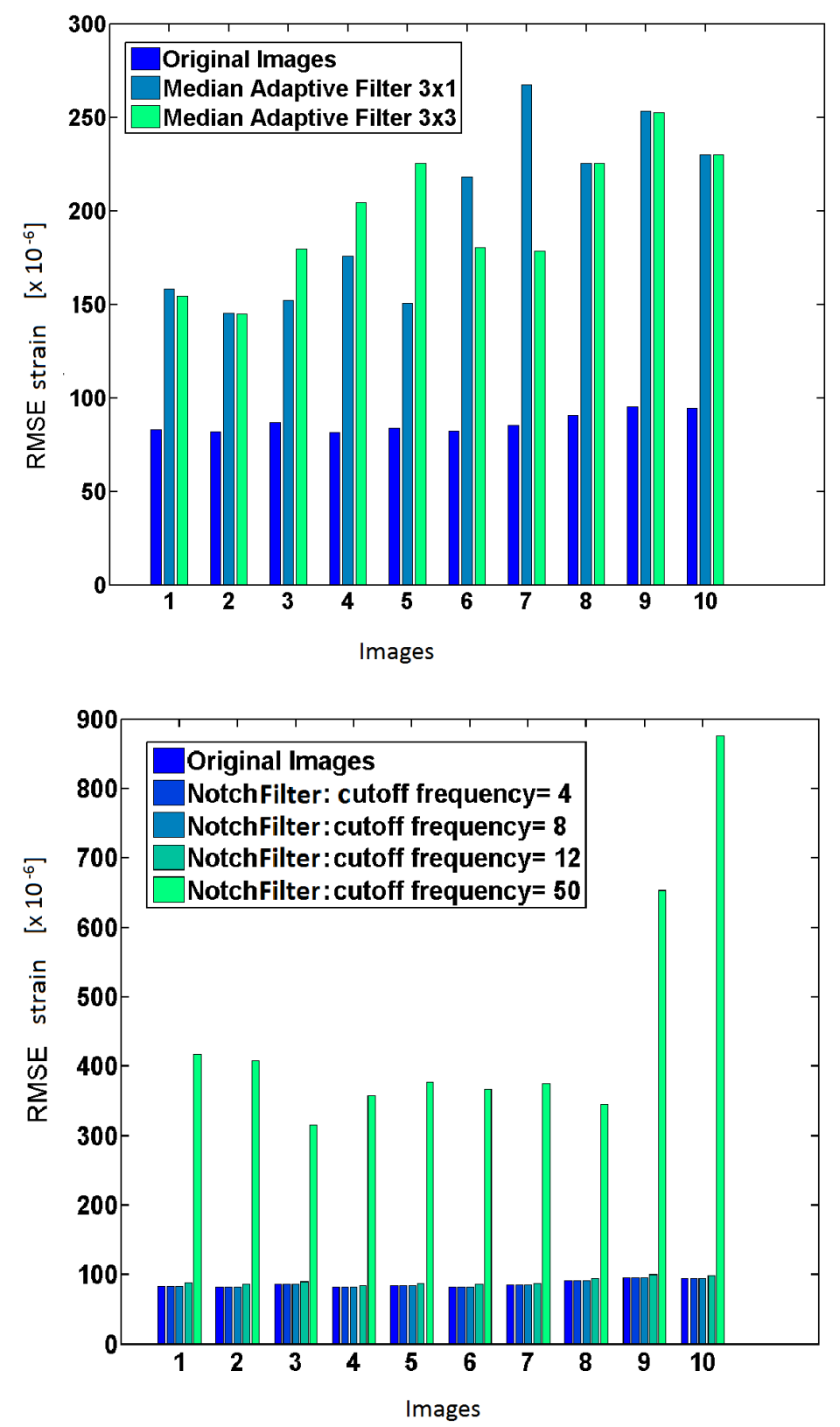

Figure 7 - Effect of filtering the input images: the RMSE of the DIC-computed $x$ component of strain is plotted for the ten acquired images (which were theoretically identical). An ideal strain distribution free from noise would have a null RMSE value. (Sub-figure a) Effect of a median adaptive filter applied to the acquired images. (Subfigure b) Effect of the Notch reject filters with increasing cutoff frequency applied to the acquired images. An increase of the RMSE values reveals an increase of noise due to filtering. 

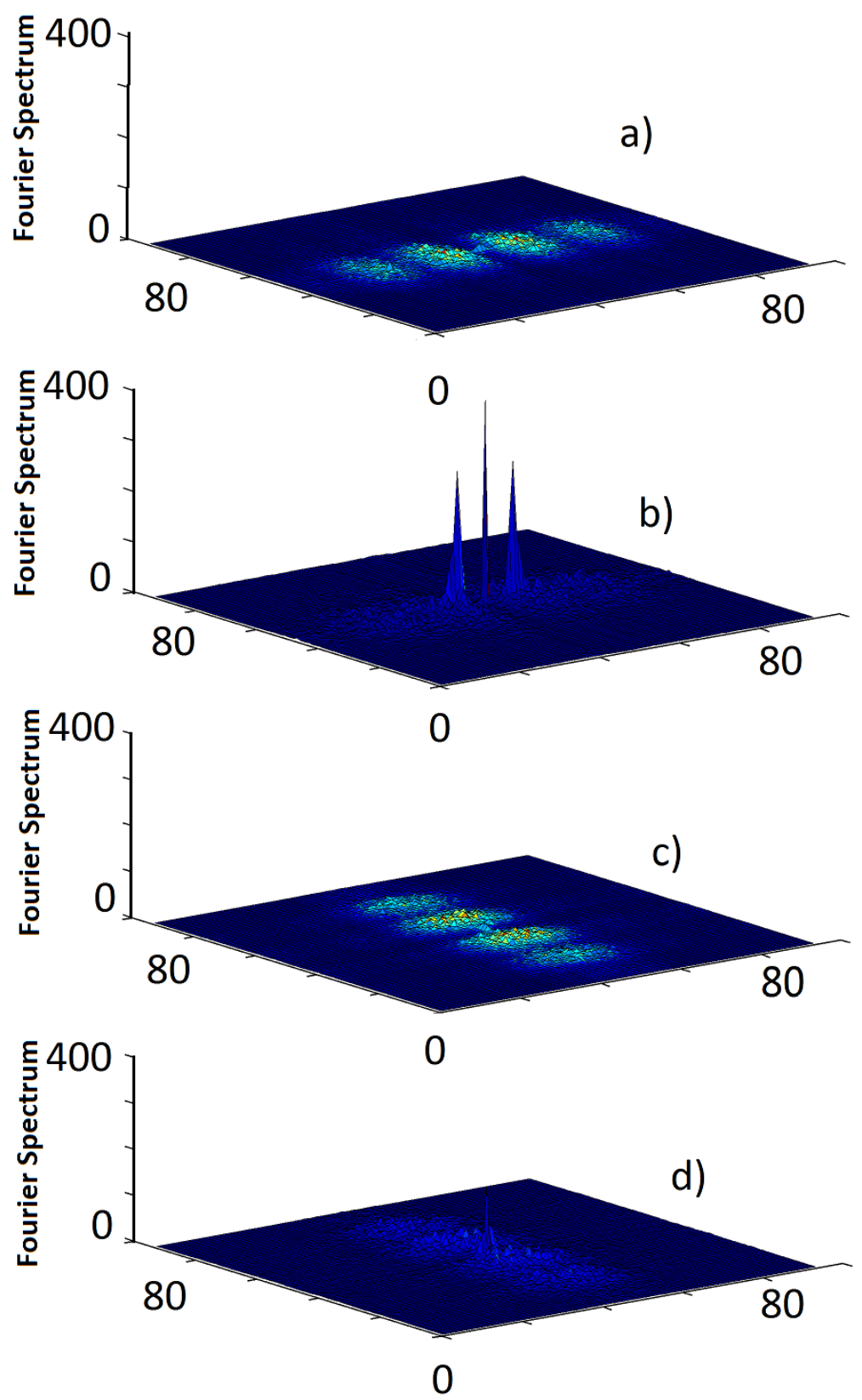

Figure 8 - Fourier amplitude spectra for the strain distributions on the cantilever test structure. Sub-figures a) and b) show the Fourier spectrum of the longitudinal component of strain (along the x-axis) for the non-strained and the strained plate respectively. Sub-figures c) and d) show the same quantities for the transversal (y) component of strain. 


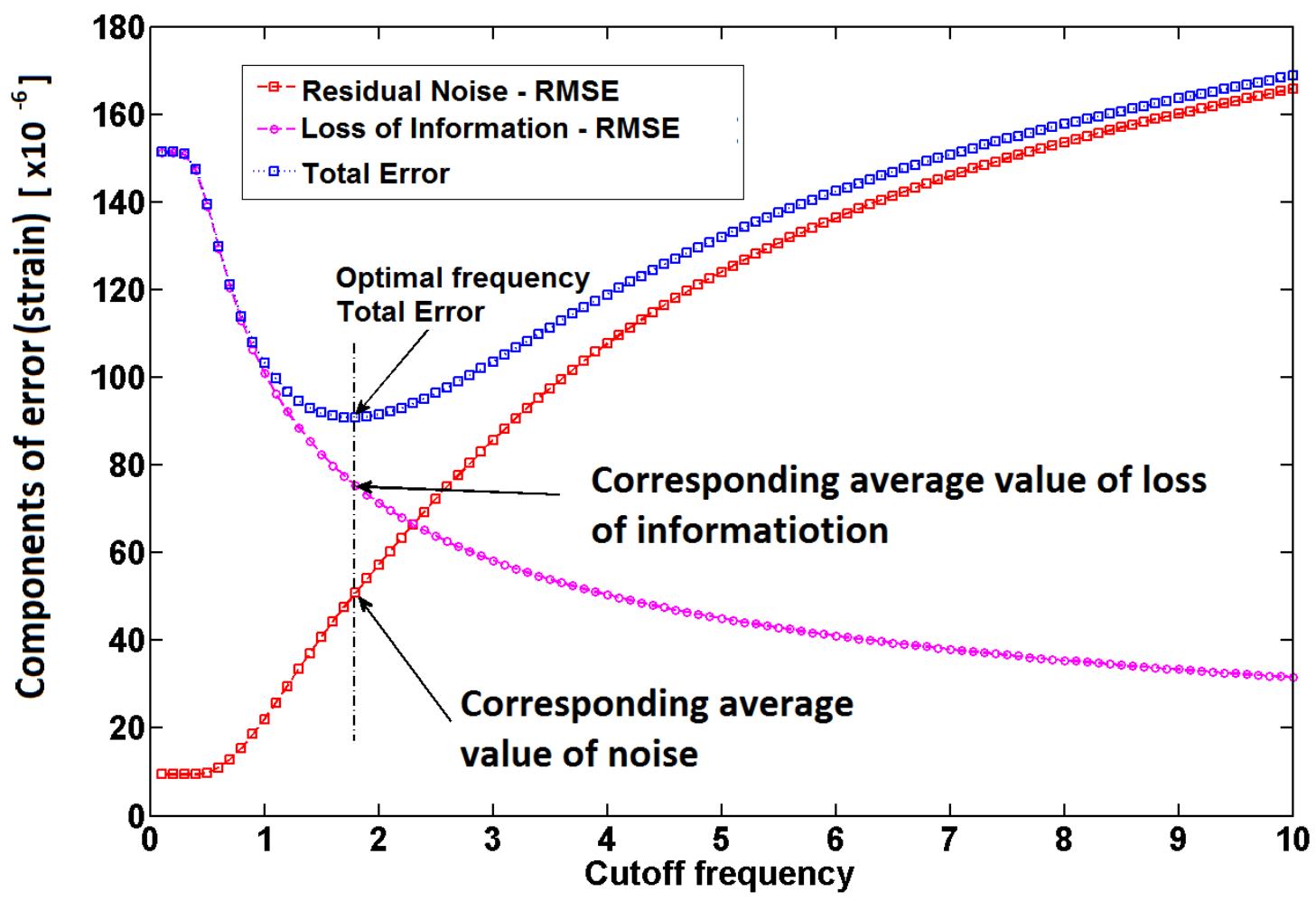

Figure 9 - Plot of the variation of the two terms composing the residual noise, loss of information and total error (which is the sum of the previous two) as a function of the cutoff frequency. The longitudinal strains in the cantilever aluminum plate were processed, when a deflection of $13 \mathrm{~mm}$ was applied at the free end of the aluminum plate (corresponding to a maximum strain of $750 \times 10^{-6}$ at the fixed end). 

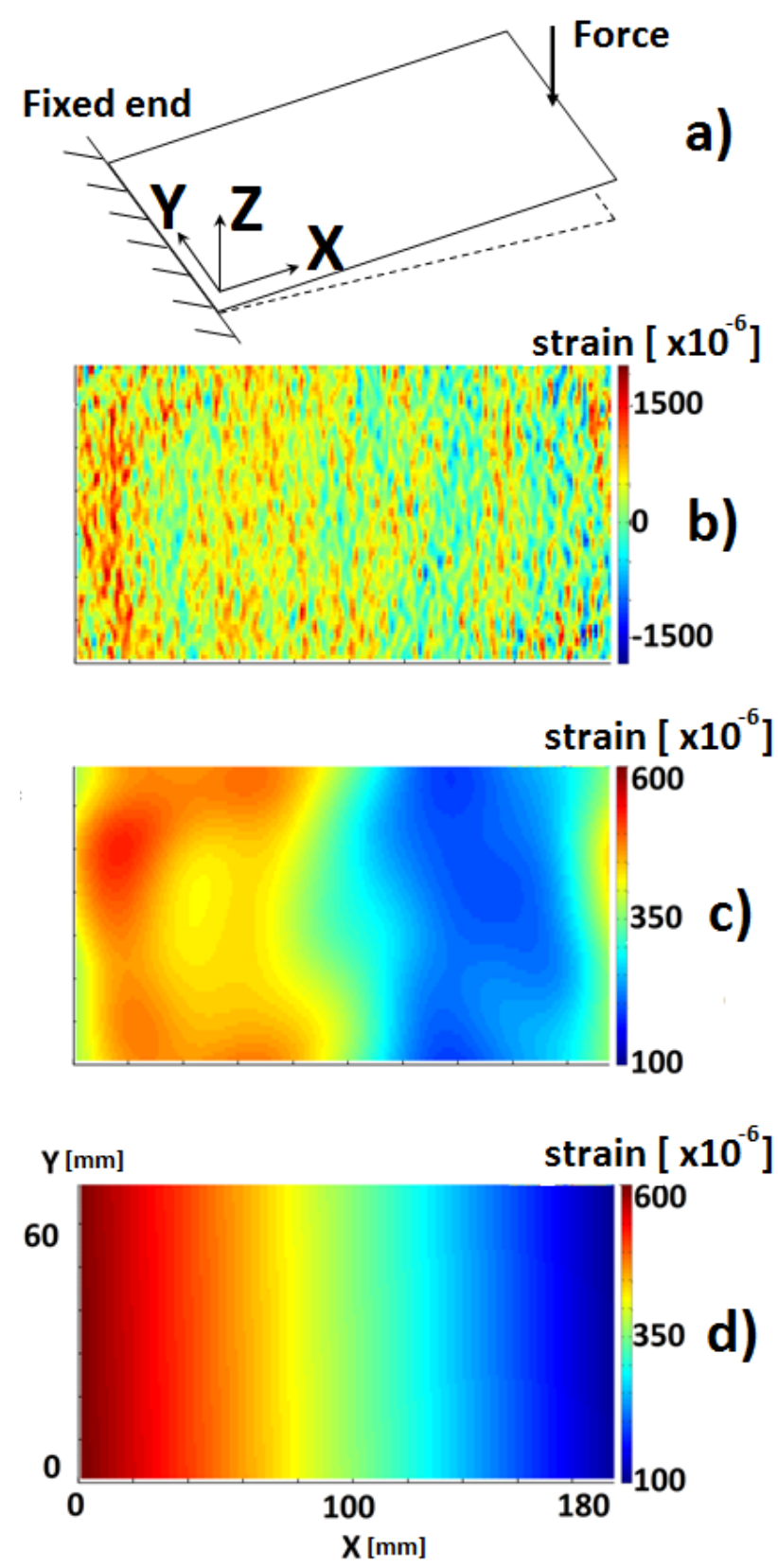

Figure 10 - Schematic of the cantilever test: a deflection of $13 \mathrm{~mm}$ was applied, inducing a maximum strain of $750 \times 10^{-6}$ at the fixed end (Sub-figure a). Unfiltered DICcomputed strain distribution (Sub-figure b). DIC-computed strain distribution after application of the optimal filter derived from Figure 9 (Sub-figure c). Theoretical strain distribution derived from the beam theory and the readouts from the seven LVTDs (Sub-figure d). 


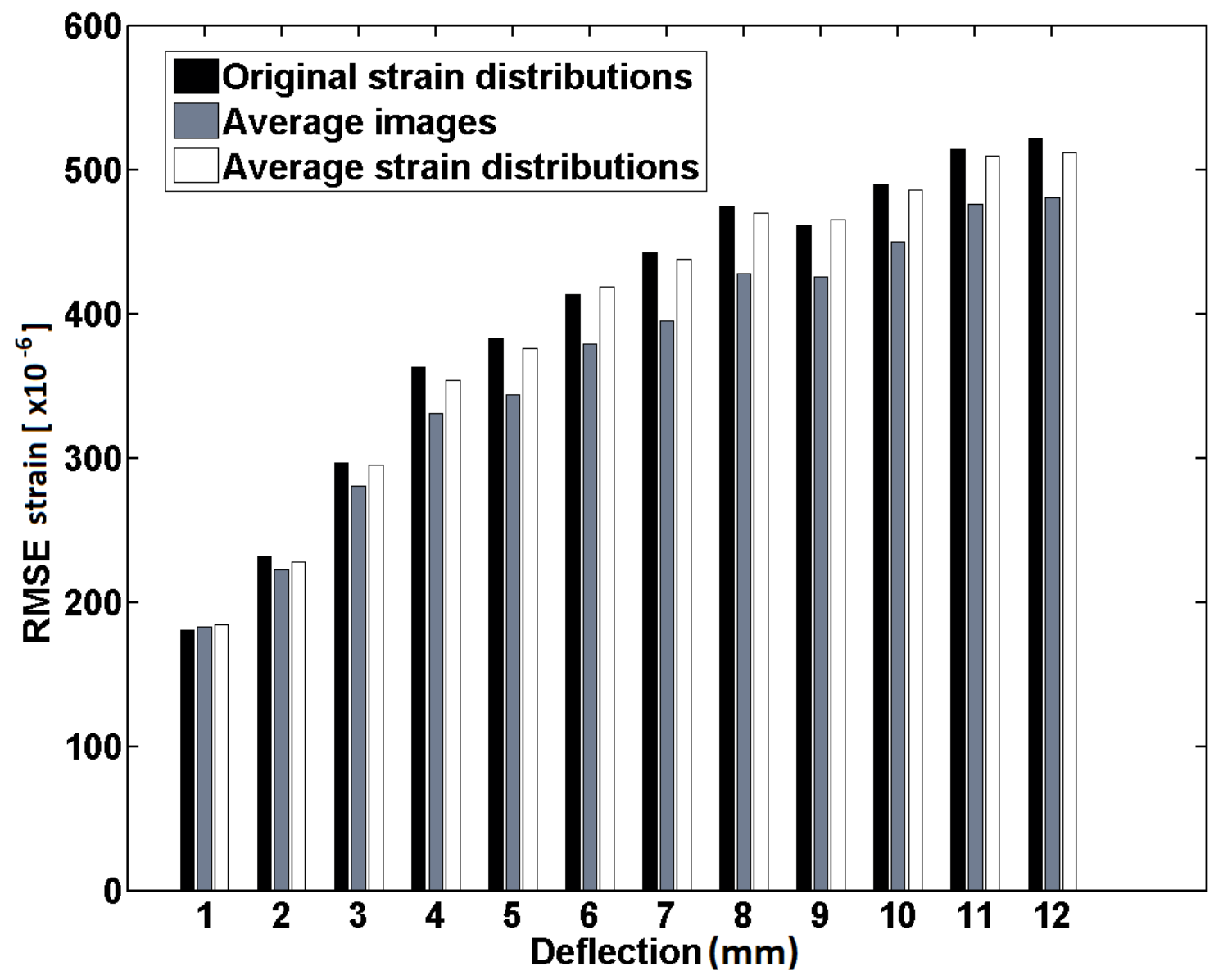

Figure 11 - Effect of filtering the acquired images and the strain distributions using image combination techniques. The RMSE values before and after the filtering are compared. While a slight decrees of RMSE was noticeable when a median combination is applied the acquired images, the same strategy applied to the strain distributions did not produce any effect. 


\section{TABLES}

Table 1 - Technical specifications of the camera used in this study (From the Baumer TGx50i vendor spreadsheet):

\begin{tabular}{|c|c|}
\hline Signal processing & real-time software programmable \\
\hline Pixel clock & $60 \mathrm{MHz}$ fast scan $/ 30 \mathrm{MHz}$ high quality (HQ) scan \\
\hline A/D converter & 14 bit \\
\hline Gain control & $0 . .20 \mathrm{~dB}$ \\
\hline Color depth & 8 bit monochrome \\
\hline Readout time & yes \\
\hline Defect pixel correction & Gigabit Ethernet IEEE $802.3 \mathrm{ab}$ with standard RJ45 connector \\
\hline Output connection & 576 Byte $($ default $) . .16$ kByte \\
\hline Stream channel packet size & $+5{ }^{\circ} \mathrm{C} \mathrm{..}+50{ }^{\circ} \mathrm{C}$ \\
\hline Operating temperature & $10 \% . .90 \%$ non condensing \\
\hline Humidity &
\end{tabular}

Table 2 - Technical specifications of the CCD sensor used in this study (ICX625ALA Sony CCD sensor vendor spreadsheet):

\begin{tabular}{|c|c|}
\hline Dark signal & $4 \mathrm{mV}\left(\mathrm{Ta}=60^{\circ} \mathrm{C}, 15\right.$ frame $\left./ \mathrm{s}\right)$ \\
\hline Dark signal shading & $2 \mathrm{mV}\left(\mathrm{Ta}=60^{\circ} \mathrm{C}, 15\right.$ frame $\left./ \mathrm{s}\right)$ \\
\hline Sensitivity & $420 \mathrm{mV}(1 / 30 \mathrm{~s}$ accumulation $)$ \\
\hline
\end{tabular}


Table 3 - Tensile test on the PTFE plate. For each of the load steps the table shows: displacement of the actuator from the initial position, theoretical average strain, DICcomputed average strain, and the corresponding standard deviation in the region of interest. The average and standard deviation were calculated by exporting the strain values of a grid of discrete points from the specimen's surface.

\begin{tabular}{|c|c|c|c|}
\hline $\begin{array}{c}\text { Actuator } \\
\text { displacement }(\mathrm{mm})\end{array}$ & $\begin{array}{c}\text { Theoretical } \\
\text { average strain } \\
\left(\times 10^{-6}\right)\end{array}$ & $\begin{array}{c}\text { DIC-computed } \\
\text { average strain } \\
\left(\times 10^{-6}\right)\end{array}$ & $\begin{array}{l}\text { Standard deviation for } \\
\text { DIC-computed strain } \\
\left(\times 10^{-6}\right)\end{array}$ \\
\hline 0.20 & 195 & 335 & 102 \\
\hline 0.40 & 287 & 465 & 192 \\
\hline 0.60 & 371 & 558 & 153 \\
\hline 0.80 & 415 & 621 & 107 \\
\hline 1.00 & 547 & 793 & 83 \\
\hline 2.00 & 1472 & 1815 & 87 \\
\hline 3.00 & 3279 & 3547 & 101 \\
\hline 4.00 & 5398 & 5735 & 96 \\
\hline 5.00 & 7297 & 7337 & 102 \\
\hline 6.00 & 8626 & 8332 & 113 \\
\hline 7.00 & 9060 & 8542 & 102 \\
\hline
\end{tabular}


Table 4 - For each of the applied strain $\left(1^{\text {st }}\right.$ column $)$, the optimal cutoff frequency to minimize the total error was computed ( $2^{\text {nd }}$ column $)$. The $3^{\text {rd }}$ and $4^{\text {th }}$ columns indicate the residual noise and the loss of information associated with such cutoff frequency, whereas the last column reports the associated total error (quadratic sum of residual noise and loss of information).

\begin{tabular}{|c|c|c|c|c|}
\hline $\begin{array}{l}\text { Strain at fixed end } \\
\qquad\left(\times 10^{-5}\right)\end{array}$ & $\begin{array}{c}\text { Optimum } \\
\text { cutoff frequency }\end{array}$ & $\begin{array}{c}\text { Residual noise } \\
\text { RMSE } \\
\left(\times 10^{-6}\right)\end{array}$ & $\begin{array}{c}\text { Loss of } \\
\text { information } \\
\text { RMSE } \\
\left(\times 10^{-6}\right)\end{array}$ & $\begin{array}{l}\text { Total Error } \\
\left(\times 10^{-6}\right)\end{array}$ \\
\hline 1.1 & 0.4 & 9 & 6 & 15 \\
\hline 3.4 & 0.5 & 10 & 11 & 21 \\
\hline 7.0 & 0.6 & 11 & 17 & 28 \\
\hline 8.6 & 0.7 & 13 & 22 & 35 \\
\hline 12.0 & 0.8 & 15 & 28 & 43 \\
\hline 13.8 & 0.9 & 19 & 30 & 49 \\
\hline 18.0 & 0.9 & 19 & 37 & 56 \\
\hline 21.2 & 1 & 22 & 41 & 63 \\
\hline 24.9 & 1.1 & 26 & 46 & 72 \\
\hline 29.8 & 1.2 & 30 & 50 & 80 \\
\hline 33.5 & 1.2 & 30 & 53 & 83 \\
\hline 37.3 & 1.3 & 33 & 54 & 87 \\
\hline 41.7 & 1.3 & 33 & 58 & 91 \\
\hline 45.1 & 1.3 & 33 & 60 & 93 \\
\hline 49.4 & 1.4 & 37 & 61 & 98 \\
\hline 53.0 & 1.4 & 37 & 64 & 101 \\
\hline 57.0 & 1.5 & 41 & 66 & 107 \\
\hline 61.8 & 1.6 & 44 & 68 & 112 \\
\hline 64.9 & 1.6 & 44 & 72 & 116 \\
\hline 68.9 & 1.6 & 44 & 73 & 117 \\
\hline 74.7 & 1.8 & 51 & 75 & 126 \\
\hline
\end{tabular}

